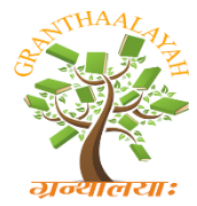

\author{
INTERNATIONAL JOURNAL OF RESEARCH - \\ GRANTHAALAYAH \\ A knowledge Repository
}

Science

\title{
THE EFFICACY OF DASHMOOLADI CHURNA IN ESSENTIAL HYPERTENSION: A CASE STUDY
}

\author{
Dr. Pravin Suresh Bhange ${ }^{1}$, Dr. Vasundhara Ramdas Borakhade ${ }^{1}$ \\ ${ }^{1}$ M.D (Scholar), Kaychikitsa Department, Yashwant Ayurvedic college, PGT \& RC Kodoli, tal- \\ Panhala,Dist-Kolhapur, India \\ ${ }^{2}$ M.D (Kaychikitsa), Kaychikitsa Department, Yashwant Ayurvedic college, PGT \& RC Kodoli, \\ tal-Panhala,Dist-Kolhapur, India
}

\begin{abstract}
Ayurveda is one of the most ancient medical sciences of the world. It describes the basic and applied aspects of life process, health, disease and its management with the sound base of principles and approaches.

21 st century is a world of industrialization \& fast life which has created various life style disorders. Hypertension is one of life threatening gift of today's life, which is psychosomatic, hereditary and occurring as a result of aging. It is also called as a silent or hidden killer because most of sufferers $(85 \%)$ are asymptomatic and as per available reports, in more than $94 \%$ cases of hypertension under lying cause is not found. Such patients are said to have Essential Hypertension (EHT). Hypertension is one of the leading causes of the Global burden of disease.

60 Years male patient suffering from Anidra, Shirashoola, Bhrama, Daurbalya, Klama, Krodhaprachurya since last two months. He came to our hospital for Ayurvedic treatment. With our unique herbal combination of drug, his all symptoms got controlled.

This is one single study on Dashmooladi churna to evaluate the efficiency on Essential hypertension.

Keywords: Dashmooladi Churna; Essential Hypertension; Case Study.

Cite This Article: Dr. Pravin Suresh Bhange, and Dr. Vasundhara Ramdas Borakhade. (2019). "THE EFFICACY OF DASHMOOLADI CHURNA IN ESSENTIAL HYPERTENSION: A CASE STUDY." International Journal of Research - Granthaalayah, 7(6), 223-229. https://doi.org/10.29121/granthaalayah.v7.i6.2019.799.
\end{abstract}

\section{Introduction}

Hypertension is one of the leading cause of the Global burden of disease. Approximately 7.6 million deaths (13-15\% of total) \& 92 million disability-adjusted life years worldwide were attributed to high blood pressure. ${ }^{1}$ The prevalence rate of hypertension increases with age. The prevalence of hypertension increases to $75 \%$ to $80 \%$ in individuals above 70 years of age. ${ }^{2}$ Genetic factors also contribute to prevalence of hypertension. Hypertension is common in Black Races, 
worldwide. Up to the age of 60 years, male individuals are more prone to the hypertension while after 60 years of age, female individuals are more prone to hypertension. Hypertension is said to be Primary/Essential when there is no obvious precipitating factor, or the much less common secondary hypertension where there is some identifiable cause. ${ }^{3}$

Our ancient Acharyas of Ayurveda explained various important properties of Herbal drugs in Samhitas \& Nighantus. With the help of those references in this topic, we are going to assess the effect of the Dashmooladi Churna in essential hypertension.

\section{Case study}

A 60 Years male patient came to us with chief complaints of -

- Anidra

- Shirashoola

- Bhrama

- Daurbalya

- Klama

- Krodhaprachurya

Patient had above complaints since last 2 months.

\section{History of past illness}

No H/o Dm/ Any other major surgery

\section{History of personal illness}

The patient was normal 2 months back, since than patient have been suffering from Anidra, Shirashoola, Bhrama, Daurbalya, Klama, Krodhaprachurya. For best Ayuvredic treatment patient came to our hospital.

\section{Personal History}

1) Ahara - mix (Vegetarian and Non- Vegetarian both)

2) Vihara - Diwaswap, sanitary life style.

3) Koshtha - Madyam

4) Agni-Vishama

5) Nidra - Anidra

6) Vyasana - Tea, Tobacco chewing.

\section{Ashtavidha-Pariksha}

\begin{tabular}{|c|c|c|c|}
\hline Nadi & $: 76 / \mathrm{min}$ & Shabda & $\therefore$ spastha \\
\hline Mala & $\therefore$ samyaka & Sparsha & : anushsn \\
\hline Mutra & $\therefore$ samyaka & Druk & : prakrut \\
\hline Jivha & : niram & Akruti & krush \\
\hline
\end{tabular}




\section{General Examination}

Pulse - 76/min

Blood Pressure - 158/90 mm/hg

Weight- $54 \mathrm{~kg}$

Prakruti - vata pradhana pittanubandhi.

\section{Material and Method}

\section{Material}

Dashmooladi Churna:
\begin{tabular}{|l|l|} 
Contents: \\
\hline 1 & Dashmool-1gm \\
\hline 2 & Shankhapushpi-500mg \\
\hline 3 & Jatamansi-500mg \\
\hline 4 & Punarnava-500mg \\
\hline 5 & Vacha-250mg \\
\hline 6 & Ela-250mg \\
\hline
\end{tabular}

Method

Preparation

- Required quantities of dravyas was taken \& fine powder formed.

- Dashmooladi Churna prepared in the dept. of Rasashastra and Bhaishajyakalpna at Ayurveda College.

\section{Standardization of Drug}

Standardization and authentication of drug material done from G.M.P. certified Company.

Route : Oral

Dose $: 3 \mathrm{Gm}$ (8 Hourly)

Anupan : Jala

Duration : 30 days

Follow up was taken on: Day 3, 5, 7, 9, 15, 21, 30.

Center of study: yashwant ayurvedic college, Kolhapur.

Type of study: Simple Randomized case study.

\section{Discussion}

\section{Nidan Panchak}

Hetu - stress, excess salt in diet, tea, tobacco chewing, ratrijagrana.

Purvarupa- Avhykta

Rupa-Anidra, Shirashoola, Bhrama, Daurbalya, Klama, Krodhaprachurya.

Upashay/anupashay — upashya with rest and medicine. 
Differential Diagnosis- Essential hypertension, secondary hypertension.

Vyadhivinishyay- Essential hypertension.

\section{SAMPRAPTI ${ }^{4}$}

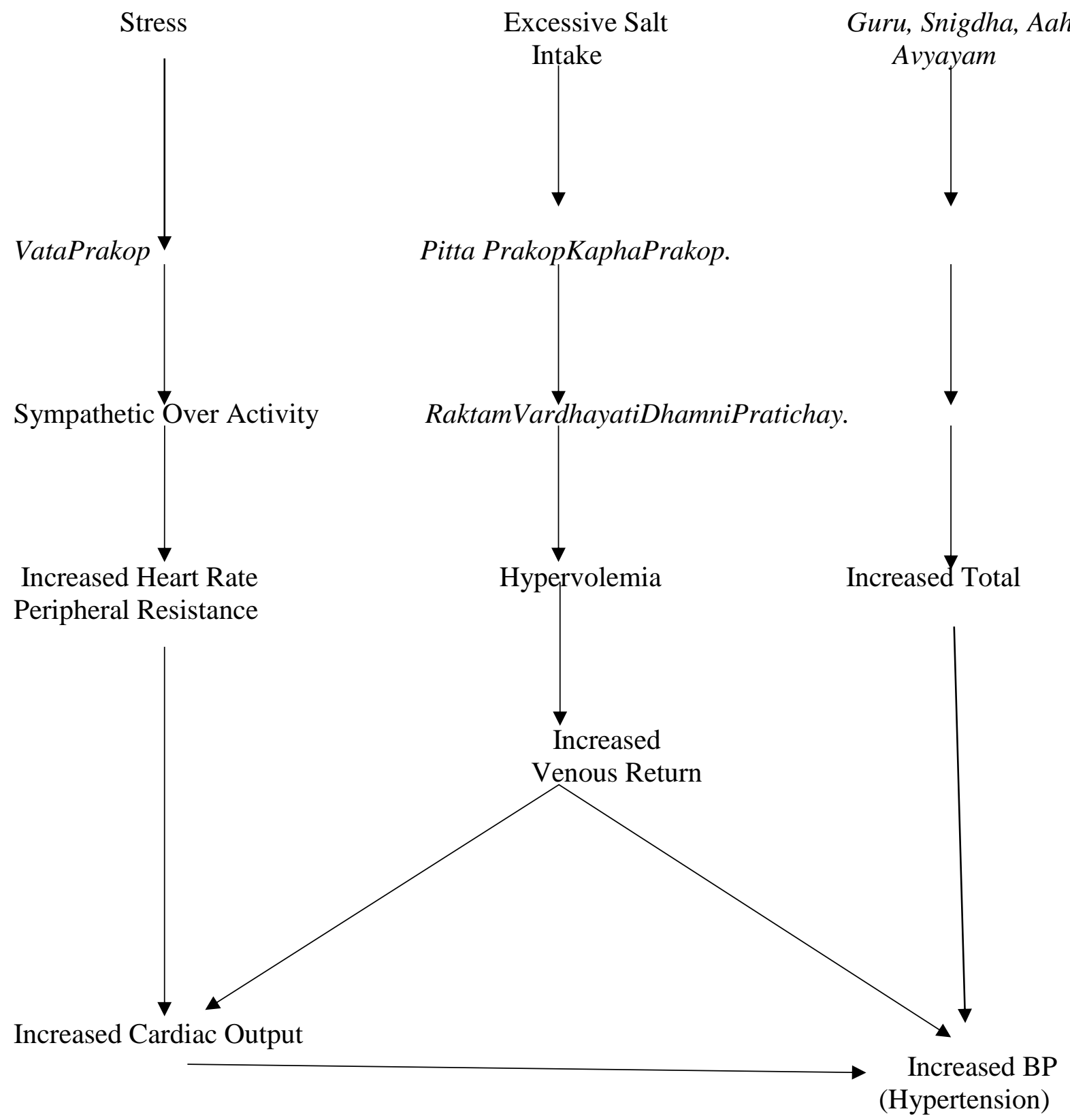

Table 1 : Showing Samprapti Ghataka

\begin{tabular}{|l|l|}
\hline Dosh & Vata, pitta. \\
\hline Dushya & Rasa, rakta. \\
\hline Stotus & Rasavaha, raktavaha, manovaha. \\
\hline
\end{tabular}


Discussion on DRUG:5,6,7,8.9

Table 2 : Showing Action of Drugs on Disease

\begin{tabular}{|c|c|c|c|c|c|c|c|c|}
\hline $\begin{array}{l}S \\
r . \\
N \\
o\end{array}$ & $\begin{array}{l}\text { Content } \\
\text { Latin Name }\end{array}$ & Guna & Rasa & $\begin{array}{l}\text { Vipa } \\
k\end{array}$ & $\begin{array}{l}\text { Vee } \\
\text { rya }\end{array}$ & $\begin{array}{l}\text { Prab } \\
\text { hav }\end{array}$ & Karma & $\begin{array}{l}\text { Upayuktan } \\
g\end{array}$ \\
\hline 1 & $\begin{array}{l}\text { Bilva-Aegle } \\
\text { Marmelos }\end{array}$ & $\begin{array}{l}\text { Laghu, } \\
\text { Snigdha, } \\
\text { Tikshna }\end{array}$ & $\begin{array}{l}\text { KatuTikta } \\
\text { Kashaya }\end{array}$ & Katu & $\begin{array}{l}\text { Ush } \\
n a\end{array}$ & & $\begin{array}{l}\text { Shothahara } \\
\text { (mutral) }\end{array}$ & Moola \\
\hline 2 & $\begin{array}{l}\text { Agnimanth- } \\
\text { PremnaSerratifoli } \\
a\end{array}$ & $\begin{array}{l}\text { Laghu } \\
\text { Ruksha }\end{array}$ & KatuTikta & Katu & $\begin{array}{l}\text { Ush } \\
n a\end{array}$ & & & Moola \\
\hline 3 & $\begin{array}{l}\text { Gambhari- } \\
\text { GmelinaArborea }\end{array}$ & $\begin{array}{l}\text { Laghu } \\
\text {,Ruksha }\end{array}$ & $\begin{array}{l}\text { Tikta,Kashaya,m } \\
\text { adhura }\end{array}$ & Katu & $\begin{array}{l}\text { Ush } \\
n a\end{array}$ & & $\begin{array}{l}\text { Shothahara, } \\
\text { Hridya,Med } \\
\text { hya }\end{array}$ & Moola \\
\hline 4 & $\begin{array}{l}\text { Patala- } \\
\text { SterosprmumSuve } \\
\text { olens }\end{array}$ & $\begin{array}{l}\text { Laghu, } \\
\text { Ruksha }\end{array}$ & Tikta,Kashaya & Katu & $\begin{array}{l}\text { Ush } \\
n a\end{array}$ & & Shothahara & Moola \\
\hline 5 & $\begin{array}{l}\text { Shyonak- } \\
\text { OroxylumIndicum }\end{array}$ & $\begin{array}{l}\text { Laghu, } \\
\text { Ruksha }\end{array}$ & $\begin{array}{l}\text { Tikta,Kashya,Kat } \\
\text { u,Madhura }\end{array}$ & Katu & $\begin{array}{l}\text { Ush } \\
n a\end{array}$ & & $\begin{array}{l}\text { Shothahara, } \\
\text { Hrudya }\end{array}$ & Moola \\
\hline 6 & $\begin{array}{l}\text { Shaliparni- } \\
\text { DesmodiumGang } \\
\text { eticum }\end{array}$ & $\begin{array}{l}\text { Guru, } \\
\text { Snigdha }\end{array}$ & Madhura,Tikta & $\begin{array}{l}\text { Madh } \\
\text { ura }\end{array}$ & Sita & & Shothahara & Moola \\
\hline 7 & $\begin{array}{l}\text { Prushniparni- } \\
\text { UrariaPicta }\end{array}$ & $\begin{array}{l}\text { Laghu, } \\
\text { Sara }\end{array}$ & Madhura,Katu & $\begin{array}{l}\text { Madh } \\
\text { ura }\end{array}$ & $\begin{array}{l}\text { Ush } \\
n a\end{array}$ & & & Moola \\
\hline 8 & $\begin{array}{l}\text { Bruhati-Solanum } \\
\text { Indicum }\end{array}$ & $\begin{array}{l}\text { Laghu, } \\
\text { Ruksha }\end{array}$ & Katu,Tikta & Katu & $\begin{array}{l}\text { Ush } \\
n a\end{array}$ & & Hrudhya & $\begin{array}{l}\text { Moola,Phal } \\
\text { a,Patra }\end{array}$ \\
\hline 9 & $\begin{array}{l}\text { Kanthakari- } \\
\text { Solanum } \\
\text { Virginianum }\end{array}$ & $\begin{array}{l}\text { Laghu, } \\
\text { Ruksha }\end{array}$ & Tikta,Katu & Katu & $\begin{array}{l}\text { Ush } \\
n a\end{array}$ & & Hrudhya & Panchang \\
\hline $\begin{array}{l}1 \\
0\end{array}$ & $\begin{array}{l}\text { Gokshuru- } \\
\text { TribulusTerrestri } \\
\text { s }\end{array}$ & $\begin{array}{l}\text { Guru, } \\
\text { Snigdha }\end{array}$ & Madhura & $\begin{array}{l}\text { Madh } \\
\text { ura }\end{array}$ & Sita & & $\begin{array}{l}\text { Mutrala,Hr } \\
\text { udhya }\end{array}$ & $\begin{array}{l}\text { Moola,Phal } \\
a\end{array}$ \\
\hline $\begin{array}{l}1 \\
1\end{array}$ & $\begin{array}{l}\text { Vacha } \\
\text { (Acoruscalamus) }\end{array}$ & $\begin{array}{l}\text { Laghu } \\
\text { Tikshna }\end{array}$ & Katu, Tikta & Katu & $\begin{array}{l}\text { Ush } \\
n a\end{array}$ & $\begin{array}{l}\text { Med } \\
\text { hya }\end{array}$ & $\begin{array}{l}\text { Mutral, } \\
\text { Medhya, } \\
\text { Hrudya }\end{array}$ & Moola \\
\hline $\begin{array}{l}1 \\
2\end{array}$ & $\begin{array}{l}\text { Punarnava(Boera } \\
\text { viadiffusa) }\end{array}$ & $\begin{array}{l}\text { Laghu } \\
\text { Ruksha }\end{array}$ & $\begin{array}{l}\text { Kasha, } \\
\text { Tikta }\end{array}$ & Katu & $\begin{array}{l}\text { Ush } \\
n a\end{array}$ & & $\begin{array}{l}\text { MutralMedh } \\
\text { ya }\end{array}$ & Panchang \\
\hline
\end{tabular}


DOI: 10.5281/zenodo.3271601

\begin{tabular}{|l|l|l|l|l|l|l|}
\hline $\begin{array}{l}\text { Ela(Elettariacard } \\
\text { amomum) }\end{array}$ & $\begin{array}{l}\text { Laghu, } \\
\text { Ruksha }\end{array}$ & Madhura,katu & $\begin{array}{l}\text { Madh } \\
\text { ura }\end{array}$ & $\begin{array}{l}\text { Shit } \\
a\end{array}$ & $\begin{array}{l}\text { MutralHrud } \\
\text { ya }\end{array}$ & Panchang \\
\hline $\begin{array}{l}\text { Shankhapushpi } \\
\text { (Convolvulus } \\
\text { pluricaulis) }\end{array}$ & $\begin{array}{l}\text { SnigdhaPi } \\
\text { chchil }\end{array}$ & Kashay & $\begin{array}{l}\text { Madh } \\
\text { ur }\end{array}$ & $\begin{array}{l}\text { Ush } \\
\text { na }\end{array}$ & Medhya & Panchang \\
\hline $\begin{array}{l}\text { Satamansi } \\
\text { (Nordostachysjat } \\
\text { amansi) }\end{array}$ & Laghu & $\begin{array}{l}\text { Madur, } \\
\text { KashayKatu }\end{array}$ & $\begin{array}{l}\text { Madh } \\
\text { ur }\end{array}$ & $\begin{array}{l}\text { She } \\
\text { et }\end{array}$ & Medhya & Moola \\
\hline
\end{tabular}

\section{Observation and Result}

Table 3 : Showing Gradiation of Symptoms

\begin{tabular}{|c|c|c|c|c|c|}
\hline $\begin{array}{l}\text { Sr. } \\
\text { No. }\end{array}$ & Lakshan & $\begin{array}{l}\text { No } \\
\text { (0) }\end{array}$ & $\begin{array}{l}\text { Mild } \\
\text { (1) } \\
\text { Rarely. } \\
\text { Relieves } \\
\text { without } \\
\text { medication }\end{array}$ & $\begin{array}{l}\text { Moderate } \\
\text { (2) } \\
\text { Frequently. Relieves } \\
\text { after sometime. Does } \\
\text { not disturb daily } \\
\text { activities. }\end{array}$ & $\begin{array}{l}\text { Severe } \\
\text { (3) } \\
\text { Frequently. Severe. } \\
\text { Disturbs daily } \\
\text { activities. Requires } \\
\text { medication. }\end{array}$ \\
\hline 1 & Anidra & & & $\sqrt{ }$ & \\
\hline 2 & Shirashoola & & & $\sqrt{ }$ & \\
\hline 3 & Bhrama & & $\sqrt{ }$ & & \\
\hline 4 & Daurbalya & & & $\sqrt{ }$ & \\
\hline 5 & Klama & & $\sqrt{ }$ & & \\
\hline 6 & Krodhaprachurya & & & $\sqrt{ }$ & \\
\hline
\end{tabular}

Table 4 : Showing Follow up According to Days

\begin{tabular}{|l|lll|l|l|l|l|l|} 
Sr. No. & $\begin{array}{l}\text { Day of follow } \\
\text { Up } \\
\text { Lakshana } \\
\text { Anidra }\end{array}$ & $\mathbf{3}$ & $\mathbf{5}$ & $\mathbf{7}$ & $\mathbf{9}$ & $\mathbf{1 5}$ & $\mathbf{2 3}$ & $\mathbf{3 0}$ \\
\hline $\mathbf{1}$ & Shirashool & 1 & 1 & 1 & 0 & 0 & 0 & 0 \\
\hline $\mathbf{2}$ & Bhrama & 2 & 2 & 2 & 1 & 1 & 1 & 1 \\
\hline $\mathbf{3}$ & Daurbalya & 1 & 1 & 0 & 0 & 0 & 0 & 0 \\
$\mathbf{4}$ & Klama & 2 & 2 & 2 & 2 & 1 & 1 & 1 \\
\hline $\mathbf{5}$ & KrodhaPrachurya & 1 & 1 & 1 & 1 & 1 & 1 & 1 \\
\hline $\mathbf{6}$ & Systolic BP & 2 & 2 & 2 & 1 & 1 & 1 & 1 \\
\hline $\mathbf{7}$ & Distolic BP & 158 & 150 & 150 & 150 & 140 & 140 & 140 \\
\hline $\mathbf{8}$ & & 90 & 90 & 90 & 90 & 90 & 80 & 80 \\
\hline
\end{tabular}

\section{Conclusion}

Ayurved has unique approach towards all disease. Since disease like hypertension has limitation in other pathy. Ayurved has best medicine without any side effect. This is single case study on 
essential hypertension. Further, I will done many more cases to evaluate efficiency of dahsmuladi churna on essential hypertension.

\section{References}

[1] kasper fauci -Harrison's Principle of internal medicine 2nd volume 19th edition Mc Graw Hill publication 2005 Hypertensive Vascular Disease Page no 1611.

[2] Godwallas Medicine for Students 24th edition -ELSEVIER publication -The Cardiovascular System -page no 227

[3] https://en.m.wikipedia.org/wiki/Essential_hypertension (27 june 2017: $10.30 \mathrm{am}$ )

[4] Y P Munjal - API Textbook of Medicine -1st volume -10th edition 2015 -Cardiology- Hypertension and its management -page no 916

[5] Dr Prakash Hegde, Dr Harini -A textbook of Dravyaguna vijnana -2nd volume -Reprint 2016

[6] Acharya Priyavat Sharma and Dr Guruprasad Sharma -Kaiyadev Nighantu'Pathyapathyavibodhak' -Reprint 2013 -Chaukhamba Orientalia -Vranasi -Page no 7,8,9,11,12,16,622.

[7] Vishwanath Dwivedi Shashtri- Bhavprakash Nighantu -Motilal Banarasidas publication -9th edition -1977page no 145,147,148,150,151,153,154,155,156,157,244,261,117,127,32.

[8] Acharya Priyavat Sharma and Dr Guruprasad Sharma-Dhanvantari Nighantu-2nd edition-Chaukhamba Orientalia publication -Varanasi-1998-page no-35,37,31,71,77,136,98,64,31,32,34,33,36.

[9] Acharya Priyavat Sharma-Priya Nighantu-2nd edition-Chaukhamba Surbharti publication -Varansi-1995-page no-8, 10, 81, 11, 12.

\footnotetext{
*Corresponding author.

E-mail address: pravinbhange07@ gmail.com/vasudha.borakhade@gmail.com
} 